\title{
Everything is Software
}

\author{
GALO CANIZARES \\ The Ohio State University
}

The world has become so inundated with software platforms, operating systems, and interfaces that the materiality of computation (circuits, electrical impulses, etc) have faded back into an unconscious layer, or a "black box." In architecture, projects rely on a wide assortment of software packages and standalone applications for design, development, and deployment, yet it's not until recently that architects have begun to reflect on the structure of those programs and how they have infiltrated our disciplinary conventions. Through examples, this paper illustrates how some designers are finding ways to talk about software theory, and proposes new methods for critically engaging with facets of software rarely discussed in design practice and education, such as the politics/biases of interfaces and the material realities of computation.

\section{INTRODUCTION}

[Digital technologies] are no longer the tools for making: they are primarily tools for thinking.

-Mario Carpo, The Alternative Science of Computation ${ }^{1}$

While it is common knowledge that computation involves electrical impulses and ones and zeroes and a lot of circuitry, most daily interactions with silicon microchips rarely require an understanding of capacitors or binary code. The inner workings of these machines are filtered through graphical user interfaces (black boxes, if you will), which shield us from the messiness of source and assembly code. And though we constantly produce and consume digital media, our dialogues with computers begin and end with software.

As disciplinary concepts, however, digital media and software are difficult to place, particularly in architecture. ${ }^{2}$ They are often conflated and taken for granted. Some even argue that 'there is no such thing as 'digital media,' there is only software." 3 Seeing as most contemporary creative work relies heavily on these intangible layers, some disciplinary anxieties could very well stem from this lack of clear definitions. For our purposes, these terms refer to the layers of interpretive interfaces that lie between a user and a computer's operating system.

But a critical vocabulary for discussing software and digital media is still lacking. It should be noted that the following discussion refers primarily to contemporary architectural discourse. Though excellent scholarship on media and computation exists, and we can trace a strong lineage of theories from thinkers like Alan Kay to Friedrich Kittler to N. Katherine Hayles (to name a few), this essay will focus on architecture and its evolving relationship with digital media. More specifically, my concern is a new generation of architects who-clearly influenced by the aforementioned theorists-are advocating for a deeper look at the substrate of our digital artifacts and instruments. ${ }^{4}$

These architects are introducing not only new types of work, but also new means for discussing that work. For instance, the recent texts, Everything is Already an Image by John May and Postdigital Materiality by Ellie Abrons are as much recalibrations of the "digital" in architecture as design propositions. Each piece establishes a critical approach for making and interpreting virtual imagery. And yet, they also appear to circumvent software and refer to computation as an abstract mechanism. It is therefore necessary to clarify nuances currently emerging as part of discussions on software-based work and ubiquitous computing.

My focus is largely on defining vague, yet often used terms, such as postdigital. Postdigital's lack of specificity in architecture warrants more scrutiny, and inevitably requires us to track its origins in new media studies in search of a clearer definition. Expanding on this clarification, we can also see that postdigital has an accomplice, which May has identified as postorthographic. The relationship between these two terms is key to any contemporary analysis of digital media. Both constitute a spectrum of attitudes prevalent in various architectural and artistic practices. At the same time, we must keep reminding ourselves that most digital artifacts are inseparable from software. This new vocabulary suggests that digital ubiquity may not necessarily signal a complete digitization of the self or provoke a struggle for intellectual dominance over mechanical tools, but instead incite a human-centric mission to better understand the critical and cultural role that screens, software, and code play in design processes.

\section{POSTDIGITAL OR POSTORTHOGRAPHIC?}

[P]ost-internet, postdigital and the new aesthetic can be taken as attempts to grapple with the immersive and disorientating experiences of computational infrastructures as they scale up and intensify.

-David M. Berry, Postdigital Aesthetics ${ }^{5}$ 


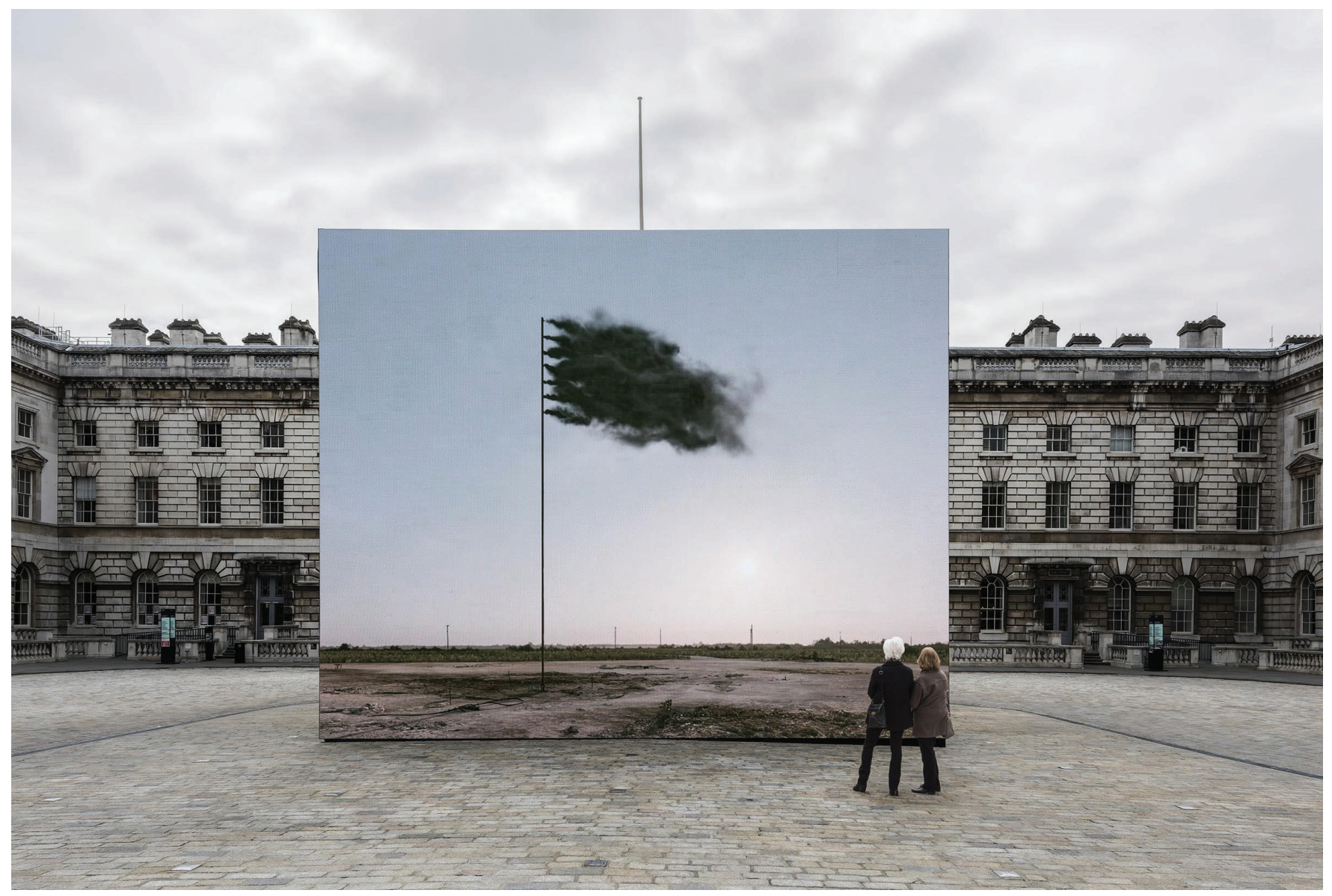

Figure 1. John Gerrard, Western Flag, 2017.

As the novelty of the digital wanes, and the word itself crumbles into a vague, dusty relic from previous unnamed futures, its attendant concepts (those of code, electric impulses, and virtual geometry) have receded into an inaccessible layer behind a wall of infinite software. This substrate, which makes up the backbone of our social, economic, and political realities, has become so pervasive that its previously accepted visualization as a set of ones and zeros is virtually obsolete. The Matrix screensaver visual has been replaced by images of data centers, stock photos of blueish gradients, clouds, and app icons, causing the word to paradoxically represent both everything and nothing simultaneously. ${ }^{6}$

In response to this collapse, two terms have emerged to rectify the discipline in their own way, postdigital and postorthographic. ${ }^{7}$ Both positions have rich theoretical underpinnings, but, for clarity, we can simply say that the former equates computation to an outsider at the service of some architectural thought, whereas the latter posits that computation has evolved into part of the thought process itself. Pragmatically speaking, we see postdigital often associated with images that do not explicitly elicit any traces of digital processes (though they are obviously digital), whereas postorthographic material very much embodies the aesthetics of data and computational logics.
Though not exactly opposites, the two terms are nevertheless polarized. Postdigital in architecture, as most recently described by Sam Jacob, comes a as melancholy reaction to the ubiquity and stylistic positivism associated with parametric design. At its core is a desire to represent certain constants from the human condition, such as materiality and narrative. In "Architecture Enters the Age of Post-Digital Drawing," Jacob points to a recovered disciplinary loss in the act of drawing out thoughts with computers. The examples put forth by Jacob and his allies of textured, saturated, flat, non-realistic images ended up equating "postdigital" with a specific style. So much so, that after its publication the consensus was that collage embodies the postdigital because it enables a "dirtier" way of working through and illustrating design narratives and concepts.

Within the digital humanities, the term postdigital aesthetics takes on a different, albeit related definition. The label is fundamentally defined as a return to low-fidelity modes of operating. Some have equated the resurgence of craft and retrograde technologies as a symptom of postdigital-ness. Others claim that postdigital enables a new understanding of materiality, one based on artistic realism and objectivity rather than analogy or abstraction. ${ }^{8}$ This results in recurring 


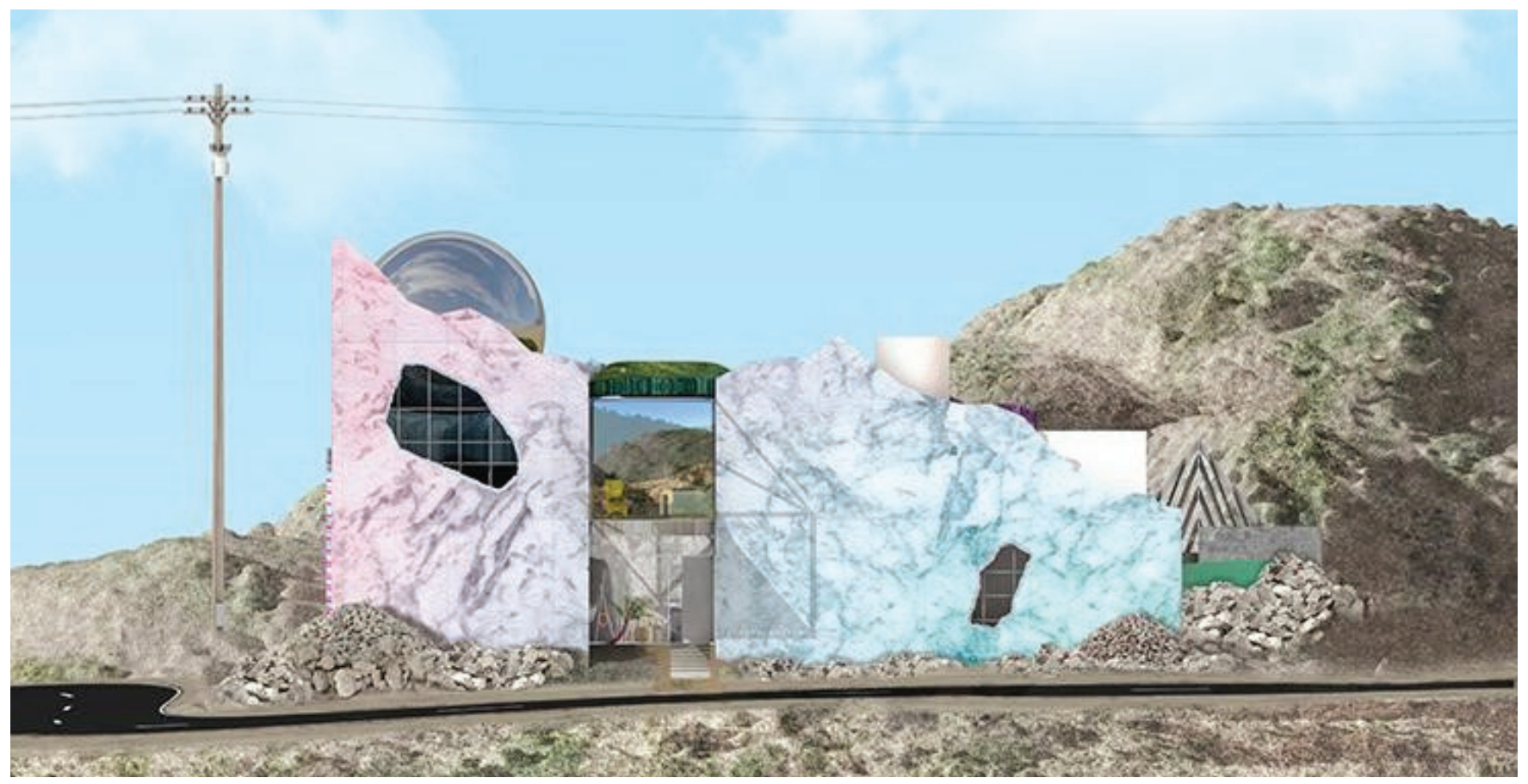

Figure 2. T+E+A+M, A Range Life, 2017.

visual tropes such as digital grain, noise, textures derived from paintings, and emulated brushstrokes. A useful definition comes from Florian Cramer's, "What is 'Post-digital'?" in which he states:

Post-digital could be understood as a moniker for a contemporary disenchantment with digital information systems and media gadgets...After Edward Snowden's disclosures of all-pervasive digital surveillance, this disenchantment has grown from a niche "hipster" phenomenon to a mainstream position that will likely impact all cultural and business practices built upon networked electronic devices and Internet services. ${ }^{9}$

In other words, it is not "post-" in the sense of after its death or exhaustion, but instead is more closely associated with postpunk or postcolonial: an ongoing cultural mutation or a rejection of the status quo that nevertheless accepts the digital as an underlying mechanism. Understanding the label as such may not provide complete immunity against being carelessly relegated to a style, but it provides a platform for further defining what, for example, postdigital architectural attitudes might be.

Postorthographic, on the other hand, expresses a more evolutionary point of view. According to May, it suggests that we no longer think in geometric terms, but rather through telematic means. While it may literally translate into after drawing, or more architecturally, "after plans, sections and elevations," in this context the term should be read as work that engages the authority of the silicon microchip. Here we see architectural media that expands and makes use of simulation, animation, automation, communication, synchronization, and visualization technologies. Postorthographic encompasses the systemic realization of "our work in 'real time,' materialized in signals and image-models." ${ }^{10}$ It also rejects the status quo, but does not linger on loss. Instead, it accepts our informatic, data-driven society, and asks how art can be extracted from such a viscous layer of convoluted systems. Those working under the umbrella of postorthographic, whether explicitly or not, use the underlying substrate of computation as a medium for critical experimentation as well as representation. The work, therefore, takes on new forms of expression, such as simulations, real-time animations, misuses of programs, bespoke software, or hacked infrastructures.

The two labels constitute a spectrum of new architectural media attitudes. Within this spectrum, everything is born in and returns to software. However, it is not a fixed distribution, since software itself allows for fluid ways of working. The contents can shift and slide, depending on their attitude towards aspects of each medium. It manifests itself as a vector map with each attitude having directional magnitudes instead of positions, and elements constantly in motion.

As an example of how to use this spectrum, let us look at materiality and consider a few contemporary practices. Here, materiality will refer to an object's "emerging event" comprised of "attention and attributes, focus, and physicality."11 This definition is key to understanding the term's ongoing mutation, as in Abrons' notion of postdigital materiality, which "flattens distinctions between physical and digital material, treating both as equally real and original." But postdigital materiality in the work 
NEW ARCHITECTURAL MEDIA TENDENCIES

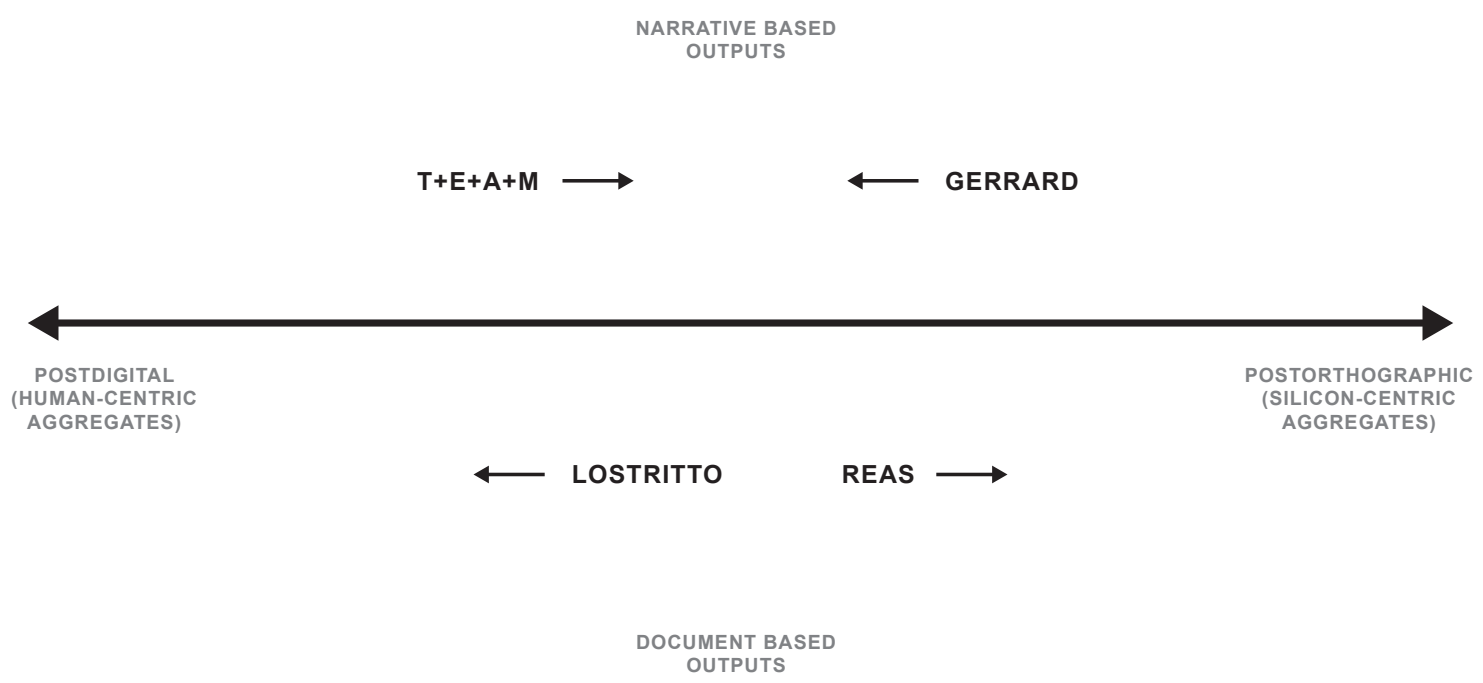

Figure 3. Chart describing the differing qualities of digital aggregates.

of $\mathrm{T}+\mathrm{E}+\mathrm{A}+\mathrm{M}$ holds postorthographic qualities, too. It requires an ability to recognize patterns in the form of computational residue, thereby prompting knowledge of specific digital processes both virtual and physical. The result of this synthesis is clearly evident in Abrons' work with $\mathrm{T}+\mathrm{E}+\mathrm{A}+\mathrm{M}$, but it also parallels the work of artist John Gerrard. Originally trained as a sculptor, Gerrard uses video-game software to produce large scale dioramas of real-world spaces. His pieces are typically sited in politically complex situations, and often allude to imbalances in contemporary power dynamics. Gerrard's most recent installation, "Western Flag" is a projected digital simulation of a flag pole that constantly emits black smoke in the shape of a flag. ${ }^{12}$ The scene is austere, yet hyper-realistic. It uses thousands of images as textures mapped on a $3 \mathrm{~d}$ recreation of the site in Spindletop, Texas. The result is so detailed that passersby might confuse the footage for a live feed of the site.

Compare this to $\mathrm{T}+\mathrm{E}+\mathrm{A}+\mathrm{M}$ 's "A Range Life" project. Materially speaking, both $\mathrm{T}+\mathrm{E}+\mathrm{A}+\mathrm{M}$ and Gerrard operate in similar ways. Both explore scenographic effects facilitated by advanced $3 d$ modeling technologies, such as texture mapping, physical light simulations, and high-fidelity meshes. Both combine site-specific components (photogrammetry) with computational processes (rendering engines) to curate new cultural associations. But where should their work be placed on our postdigital-postorthographic spectrum? I would argue that both practices converge towards center. The complexity of the material effects built from equal parts simulated particles and captured images demonstrates a desire to blend both physical and virtual assemblages to produce something else. At the same, the projects never relinquish the authority of advanced graphics engines.
If $\mathrm{T}+\mathrm{E}+\mathrm{A}+\mathrm{M}$ and Gerrard present a case for convergence, then the individual work of Carl Lostritto and Casey Reas are examples of divergent practices on the same spectrum. Lostritto and Reas could both be categorized as generative artists operating primarily in two-dimensional media. Their work is assembled and compiled as code, and usually results in large format line drawings. Despite their similarities, however, I maintain that Lostritto leans towards the postdigital, while Reas is very much postorthographic. Lostritto's body of work is a die-hard examination of the architectural drawing. From early work on pen-plotting, to a recent series of animated drawings, the line itself has been Lostritto's primary concern. ${ }^{13}$ In a way, we could interpret the emphasis on technique and control over tools as a traditional architectural pursuit, one in which anachronous forms of representation are revisited through an appropriation of software and code. "Computing/Drawing With a Vintage Pen Plotter," for instance, catalogs an ongoing series of line drawings produced with the earliest tool for materializing a computer drawing. These artifacts combine the entropic quality of ink on paper, the precision of traditional projective drawing, and the methodical act of delineating space, all retrograde characteristics of the postdigital. Reas's work, by contrast, touches on the very makeup of digital drawings: the projected pixel. The series "Still Life" deconstructs an abstract composition of 3D objects based on each pixel's HSL (Hue, Saturation, Lightness) and RGB (Red, Green, Blue) values. Reas, responsible for co-authoring the programming language, Processing, creates standalone software to automate the real-time assembly of pixels on the screen. ${ }^{14}$ Like Lostritto's 
drawings, Reas's operations result in abstract animations and compositions, yet the core of the work remains different. Whereas the former adheres to a specific disciplinary motive, the latter prioritizes a kind of postorthographic materiality, a set of effects that can only emerge from new assemblages of pixels, vectors, polygons, textures, code, data-sets, executables, and software in real-time.

Though pen-plotter drawings and 3D textured models of rocks are all digital artifacts, each embodies a different approach to materiality. One combines a hand-held tool with computation to achieve a specific texture on a mechanically controlled line, and the other creates a data-set of points in virtual space that replicate naturally occurring variation. If entropic attributes are a key component of most definitions of materiality, we can no longer ignore the inherent entropy embodied in digital aggregates. Abrons' definition of material as "stuff that's assembled and manipulated to produce things" is a much needed broadening of the term..$^{15}$ The goal of the postdigital-postorthographic spectrum is therefore to clarify the diverse attitudes towards software and digital technologies as these definitions evolve.

\section{ARE WE (EPISTEMICALLY) DIGITAL YET?}

Minesweeper is more addictive than Solitaire. Players are pitted against themselves, trying to beat their own best times. People have been known to dream about it.

\section{-The Washington Post, $1994^{16}$}

Despite efforts by operating system designers to have us dream of their software, we probably haven't become digital until recently. During the better half of the 1990s and the early 2000s, architecture operated through digital means analogically. Meaning that CAD paper-space digitized drafting tables and a common phrase thrown around was, "the tool is only as good as the designer." For architects, becoming digital referred to pragmatically adopting a software-based workflow from conceptual design to construction administration, yet always through an analogical lens. Architectural work continued to operate as it did on paper (i.e. orthographically), only with a virtual pen and virtual paper. This was further reinforced by the skeuomorphism employed by user interface designers, who proposed familiar imagery in the form of virtual icons as means of transitioning toward the digital. Of course, none of this is revelatory except to say that we may not have been epistemically digital until we broke away from these analogical workflows.

A key contributor to this evolution stems from the realization that analog need not be opposed to digital. In fact, the two could be seen as fundamentally interrelated, if not dependent

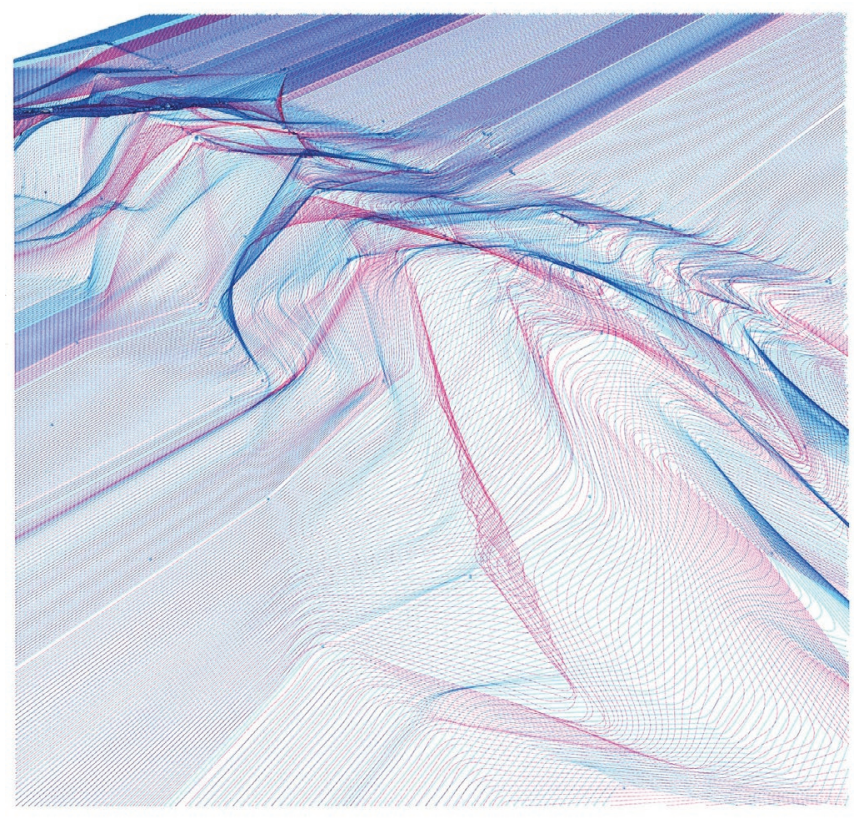

Figure 4. Carl Lostritto, K-003-070, (date unknown).

on each other. For instance, one definition of analog is: a set of information that exists continuously along various scales, such as a sound wave. This applies to the continuous electrical currents in microchips or the magnetic field of a hard drive. By contrast, if we take digital to mean, "discrete units of countable information," cellulose cinematic film may be considered digital due to its reliance on still frames and timecode. Additionally, Florian Cramer reminds us that "an LCD screen is a hybrid digital-analog system: its display is made of discrete, countable, single pixels, but the light emitted by these pixels can be measured on an analog continuum. ${ }^{17}$ Nevertheless we should be careful not to let the scale of this distinction get out of control. After all, as Friedrich Kittler mentions in "There is No Software," once we dive into the mechanisms of electrical impulses, it all fizzles into thermodynamics. ${ }^{18}$ What we might ask instead is: how does John May's definition of image as the output of energetic processes represented in discrete, measurable units, or "data," depict new ways of contemplating architectural work? ${ }^{19}$

May suggests architects are finally pushing past analogical thinking thanks to widespread software such as BIM (Building Information Modeling). It has replaced drawings with images, geometry with statistics, syntax with source code, and tectonics with commands. ${ }^{20}$ It has also skewed our priorities towards the management of information, and seduced us with seamless, real-time, synergy. Today, as workdays fill up with synchronization rituals, Googling error messages, and resetting file permissions, we may finally call ourselves digital. ${ }^{21}$ But this is only half the story. 


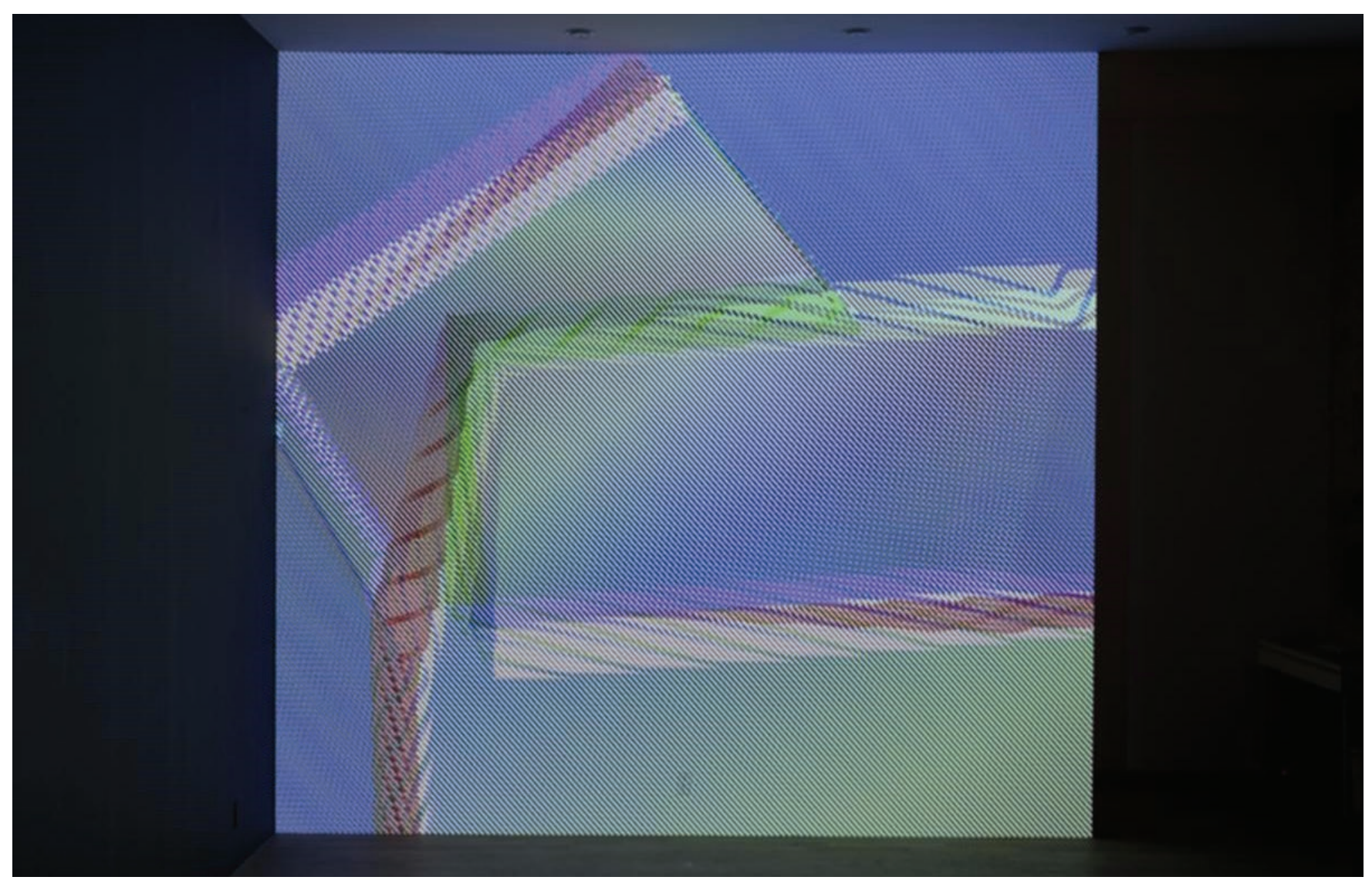

Figure 5. Casey Reas, Still Life RGB, 2016.

In order to fully grasp the significance of this shift, software must be discussed. If becoming digital involves trading in orthography for telematics, then the software collating and interpreting those signals dictates how designers interact with data. Therefore, architecture's fate may be more dependent on redundancy protocols in Autodesk's Cloud servers or security settings within a file than on relationships between pixel color values and their appearance. If everything is already an image, then the media through which we consume/store/process those images warrants as much scrutiny as the bits, bytes, electrical impulses, brightness values, and real-time updates constituting that image. We must first acknowledge that everything is not only images, but images linked to software. And that now, as Lev Manovich put it "is, therefore, the right moment to start thinking theoretically about how software is shaping our culture, and how it is shaped by culture in turn."22

What we need is an embrace of the critical and cultural effects of ubiquitous computing and software on the discipline of architecture. ${ }^{23}$ Architects are neither cyborgs nor programmers, but living in a world of ubiquitous software implies that our primary mode of communication, the architectural drawing, is a mostly digital artifact. It comes in PDF, DWG, DXF, JPG, PNG, RVT, and many other variants. The relationship between software and file formats dictates that a document's mutability hinges on a layer of code for interpretation and another for representation. The user may manipulate the file in various ways, using commands, code, or mostly likely graphical interface buttons. However, any discussion on architecture's software tends to be regarded as taboo. Architects are masters of abstract principles. They communicate their work primarily in terms of diagrams, methods, or relationships; rarely as part of a specific workflow between programs. Workflows are secret, personal devices deployed strategically. ${ }^{24}$ And yet, as the new landscape of available software and apps increases, so does the number of managerial decisions to make regarding our products.

Beyond telematics and electrical signals, these new interactions enrich the dialogue between human and machine. A user is euphoric when she discovers a new shortcut; another might be frustrated to find their changes were not saved. A client might request the full RVT file from their architect alongside the compiled construction documents. Thus, the digital architect, one who has become digital, is a figure whose principal task is not only to translate between drawing and building, but also to translate across a vast, ever-updating landscape of standardized file-types and graphical user interfaces, simultaneously making decisions about users, permissions, and longevity. ${ }^{25}$ 
What software has engendered on one hand is a kind of medium specificity in design in which software's inherent qualities dictate the aesthetics of its products (its materiality), and on the other a re-figuration of the digital's relationship to the analog. ${ }^{26}$ While the first digital turn led to a set of codified norms concerning production of digital objects, these attitudes usually regarded software as a tool in the service of higher intellect. As the variety and prowess of tools available to architectural designers has increased, software has evolved, too. Recall, for a moment, Casey Reas' standalone executable artworks. These pieces do not require a media player or image viewer, the software is the artwork. They are liberated from the constraints of third-party applications in a way that even common JPG files, which require interpreting software, are not. Software is no longer a simple vehicle for communicating that which we blindly create in our heads, but rather, much like analog media, it contributes to the formulation of that very thought from our first encounter with it, sometimes existing as the idea itself.

The above discussion of postdigital and postorthographic tendencies provides new frames of reference derived from new media art practices, but also illustrates a need for critical dissection of architectural media. A decade ago, work from designers and artists like $\mathrm{T}+\mathrm{E}+\mathrm{A}+\mathrm{M}$, John Gerrard, Casey Reas, and Carl Lostritto would all be lumped under computer art. Now that we all work on computers, we all capture images, and we're all scrolling, swiping, clicking beings, we require more terminological specificity to orient ourselves within this new playing field. May identified becoming digital as "establishing meaningful expression within imaging itself, all the while acknowledging that our images no longer mean anything at all." ${ }^{27}$ This paradox of extracting meaning out of meaningless data is perhaps where we have arrived with image sharing apps. On Instagram, for instance, users publish and consume images ceaselessly. At the time of writing, there are $71,452,378$ images catalogued under the hashtag architecture. While it is unlikely that Instagram itself will host a meaningful discourse on imaging or architecture, it has already proven to be one of the most culturally significant pieces of software. Its attendant social phenomenon has even triggered new scholarship within the digital humanities. ${ }^{28}$ Architecture, for now, moves at a slower pace. But if a piece of software aimed at sharing digital images is shaping social behaviors and contributing to a shared cultural history, then architecture, which is informed by those behaviors and histories, should acknowledge this new consciousness.

\section{ENDNOTES}

1 Mario Carpo, "The Alternative Science of Computation," e-flux Architecture (June 2017) accessed June 30, 2017.
2 In the context of this essay, software will be understood as the layer of interpretive interfaces that lie between a user and a computer's operating system. The term digital media will be used to refer to the outputs of this layer, the products of users' interactions with software.

3 There are no images without image-viewers, no MP3s without MP3 players, etc. See Lev Manovich, Software Takes Command (London: Bloomsbury Academic, 2013), 152.

4 John May's piece, "Everything is Already an Image," in Log 40 is now a de-facto reference in any conversation concerning digital images, renders, or drawings. See "Everything is Already an Image" in Log 40 (2017). Ellie Abrons has a seminar/workshop entitled "Becoming Digital" at the University of Michigan in which students unpack computation as a background condition of our reality. See "Becoming Digital," syllabus, University of Michigan, Ann Arbor, MI, 2017; and "Postdigital Materiality," in Lineament, eds., Gail Peter Borden and Michael Meredith (New York: Routledge, 2017).

5 David M. Berry and Michael Dieter, "Thinking Postdigital Aesthetics," in Postdigital Aesthetics: Art, Computation, and Design (New York: Palgrave Macmillan, 2015), 4

6 Observations on this digital $=\mathrm{cool}$ blue have been made by Florian Cramer as well as John May. See May's lecture at Harvard's Graduate School of Design: Harvard GSD, "Newish Media: A Conversation with Lucia Allais and John May," YouTube video, 1:57:52, April 11, 2017. https://www.youtube.com/ watch?v=gqCUh16R4yw

7 For post-digital see Sam Jacob “Architecture Enters the Age of Post-Digital Drawing," in Metropolis 36, no. 8 (March 2017). For post-orthographic see May, "Everything is Already an Image."

8 Ellie Abrons, "For Real" Log 41 (2017): 68.

9 Florian Cramer, "What is Post-Digital?" in Postdigital Aesthetics: Art, Computation, and Design, eds., David M. Berry and Michael Dieter (New York: Palgrave Macmillan, 2015)

10 May, "Everything is Already an Image," 19.

$11 \mathrm{~N}$. Katherine Hayles and Todd Gannon put forth this definition as it allowed them to scrutinize new media such as CD-ROMs, software, and virtual artifacts. See "Virtual Architecture, Actual Media," in The SAGE Handbook of Architectural Theory, eds., C. Greig Crysler, Stephen Cairns, and Hilde Heynen (London: SAGE Publications, 2012), 486.

12 "Western Flag (Spindletop, Texas) 2017," John Gerrard official website accessed Jan 8th, 2018. http://www.johngerrard.net/western-flag-spindletoptexas-2017.html.

13 See "Story of the Line," "Computational Hatching," and "When the Drawing is Moving," Carl Lostritto official website, accessed January 8, 2018. http:// lostritto.com/.

14 "Still Life (RGB-AV A)," 2016, REAS official website, accessed Jan 8th, 2018. http:// reas.com/still_life_rgb_av_a/.

15 Abrons, "For Real," 68

16 Joel Garreau, "Office Minefield," The Washington Post, March 9, 1994

17 Cramer, "What is Post-Digital."

18 Hayles and Gannon, "Virtual Architecture, Actual Media," 487.

19 May, "Everything is Already an Image," 12

20 May, 26.

21 Abrons' project, "Becoming Digital" asks students to critically engage computation as a "background condition of our reality." They experiment with $3 d$ scanning, internet media, and Google's Deep Dream.

22 Manovich, "Introduction," in Software Takes Command, 20.

23 Here I have extended Abrons' description of "Becoming Digital" to explicitly mention software.

24 For instance, MOS has written their own software, but never released it to the public, as far as I know. See Michael Meredith and Hilary Sample, Everything All at Once: The Software, Film, and Architecture of MOS (New York: Princeton Architectural Press, 2012).

25 Greg Lynn illustrates the problem of curating and archiving digital media in Archaeology of the Digital. He states, "very few, if any, architecture collecting institutions hold digital files in their native design format for research or exhibition." See "The End of 'In the Future'," in Archaeology of the Digital (Berlin: Sternberg Press, 2013), 13

26 See all exhaustive references to Clement Greenberg, "Modernist Painting," accessed November 1, 2017. http://www.sharecom.ca/greenberg/ modernism.html.

27 May, "Everything is Already an Image," 25.

28 Lev Manovich has self-published parts of an ongoing project on Instagram, data science, and art history. See Instagram and Contemporary Image. Accessed Jan 8, 2018 http://manovich.net/index.php/projects/instagram-and-contemporary-image. 